\title{
Exploration Challenges: Transferring Ground Repair Techniques to Space Flight Application
}

\author{
Carole A. McLemore ${ }^{1}$, James P. Kennedy ${ }^{2}$, Frederick A. Rose ${ }^{3}$, Brian W. Evans ${ }^{2}$ \\ ${ }^{I}$ Exploration Systems Development Office, NASA Marshall Space Flight Center, VP33, Huntsville AL 35812, USA \\ ${ }^{2}$ Teledyne Brown Engineering, 300 Sparkman Drive, Huntsville AL 35805, USA \\ ${ }^{3}$ bd Systems, 600 Boulevard South SW \# 304, Huntsville AL 35802, USA \\ ${ }^{1} 256.544 .2314$, carole.a.mclemore@nasa.gov
}

\begin{abstract}
Fulfilling NASA's Vision for Space Exploration will demand an extended presence in space at distances from our home planet that exceed our current experience in space logistics and maintenance. The ability to perform repairs in lieu of the customary Orbital Replacement Unit (ORU) process where a faulty part is replaced will be elevated from contingency to routine to sustain operations. The use and cost effectiveness of field repairs for ground based operations in industry and the military have advanced with the development of technology in new materials, new repair techniques and new equipment. The unique environments, accessibility constraints and Extra Vehicular Activity (EVA) issues of space operations will require extensive assessment and evolution of these technologies to provide an equivalent and expected level of assurance to mission success. Challenges include the necessity of changes in design philosophy and policy, extremes in thermal cycling, disruptive forces (such as static charge and wind entrainment) on developed methods for control of materials, dramatically increased volatility of chemicals for cleaning and other compounds due to extremely low pressures, the limits imposed on dexterity and maneuverability by current EVA equipment and practices, and the necessity of unique verification methodology. This paper describes these challenges in and discusses the effects on the established ground techniques for repair. The paper also describes the leading repair methodology candidates and their beneficial attributes for resolving these issues with the evolution of technology:
\end{abstract}

Keywords: Technology development, repair technologies, maintainability, design for maintainability, repairability, Vision for Space Exploration

PACS: $89.20 . \mathrm{Bb}, 07.87 .+\mathrm{v}$

\section{INTRODUCTION}

The In Situ Fabrication and Repair (ISFR) Element was established at the Marshall Space Flight Center (MSFC) in 2004, in response to the President's announced Vision for Space Exploration (VSE), to facilitate the goals of that Vision. The Element was established to evaluate the feasibility and to conduct the developments necessary to fabricate parts and tools at the location where the equipment is operating (i.e., in situ), using provisioned or local resources, to fabricate structures on the surface of the Moon and Mars using local resources (with or without additional provisioned materials), and to repair equipment and parts in situ utilizing non-destructive evaluation (NDE) techniques to the maximum possible extent. The scope of the activity included all mechanical, electrical and some biological components and assemblies, resulting in a very broad set of capabilities to be evaluated (Bodiford, et. al., 2005).

Because of that breadth of scope, three sub-elements were established to look in detail at the areas of Surface Structures, Fabrication of tools and parts, and Repair and NDE. Each of these areas was driven by a need to reduce necessary upmass for missions and by the need to be self-sufficient on the way to and on the surface of the Moon and Mars due to the distance from earth and the time required in transit. In situ fabrication of tools and parts would obviate the need for provisioning massively redundant spares (as well ás providing the capability to fabricate hardware for unforeseen needs) (Hammond, et. al., 2006). The ability to fabricate surface structures (wholly or partially) from in situ materials provides an enormous mass savings by reducing or eliminating the need for 
launching the structures themselves; only the construction equipment and infrastructure requires launching from Earth. Non-Destructive Evaluation was originally included in the scope because it was recognized that analysis of critical parts fabricated or repaired in situ could be necessary before they are deemed fit for use in a manned space environment, but the scope was expanded as it was recognized that other uses of NDE would enhance safety and reliability of systems and missions. Repair capabilities were included, again, to greatly reduce the necessary provisioning of spares, leading to cost savings and more upmass availability for science payloads and other necessary items.

In manned space flight, the traditional approach has been to design and build multiple-fault tolerant (redundant) systems that can operate with failed parts when reliability was an uncertainty, or to utilize an Orbital Replacement Unit (ORU) process whereby replacements are made at a very high level. This strategy has served primarily to limit crew time required to restore functionality to the damaged hardware. However, for the long-duration missions envisioned in the VSE, logistics associated with a stockpile of replacement parts will make this sort of approach impractical. Reliability will become a greater driver for extended operations component designs in lieu of fault tolerance as the loss of redundant systems is inevitable. Repair down to the component level will have to become ordinary to sustain operations.

In the area of Repair and NDE, many capabilities were evaluated and an initial down-selection made. Because the goal was to assist the development of technologies to accomplish these aims, a "first priority" capability set was selected that would provide the broadest range of usefulness in the eventual long-duration space missions, while also providing benefit in the near term, with a minimal development cost. Within each of those selected capabilities, a detailed examination of the underlying technologies was conducted, the technologies were then scored in their ability to deliver the desired capabilities, and finally recommendations were made for certain technologies to be funded for further development.

Considering that technology development budgets were limited, and in order to minimize risk, technologies with relatively high Technology Readiness Levels (TRLs) (Mankins, 1995) were favored. Many of the technologies recommended were well-proven for ground-based applications; the challenge lies in adapting their suitability to manned space flight missions and proving that the technology will work in that environment. In a word, reinvention, rather than invention of old capabilities is the key to a cost effective and efficient development program for these specific space needs. Technology infusion plans were made for integrating each of these techniques into the overall NASA Constellation Program, the program responsible for returning man to the Moon, blazing the trail to Mars and beyond. Though extensive experience is available in industry and the military testifying to the usefulness and cost effectiveness of field repairs, modifications (the aforementioned "reinvention") will have to be made to some of the technologies, and new thought processes will be needed in the design of equipment to "build in" repairability and sustainability capabilities. In designing those technology development plans, several common challenges were noted that must be addressed by any program seeking to provide technologies to meet these needs. Among these special challenges are the need to affect design philosophy and the need to design for large thermal variations, very low pressure situations, to adequately control materials and for the limited dexterity of crewmembers, and the implementation of an appropriate verification program.

\section{DEFINING CONCEPTS OF OPERATIONS}

To be able to assess what specific repair capabilities would be required in future Exploration missions, and thus what technologies were candidates for development, it was first necessary to design reference scenarios whereby those repair capabilities would be utilized. These concepts for operation were created at multiple levels, and two examples are presented here. The broad operational scenario for a repair process is depicted in Figure 1. The concept of operations for the performance of this task will need to implement all of the precautions and approvals associated with a safe mission procedure. Even though the crew will be isolated via distance they will not be without ground support in the way of communications with process and materials engineers to evaluate options and provide recommendations for standard or new process repairs. History has shown that the ground and flight teams are inseparable in assessing, dispositioning and resolving anomalies. 


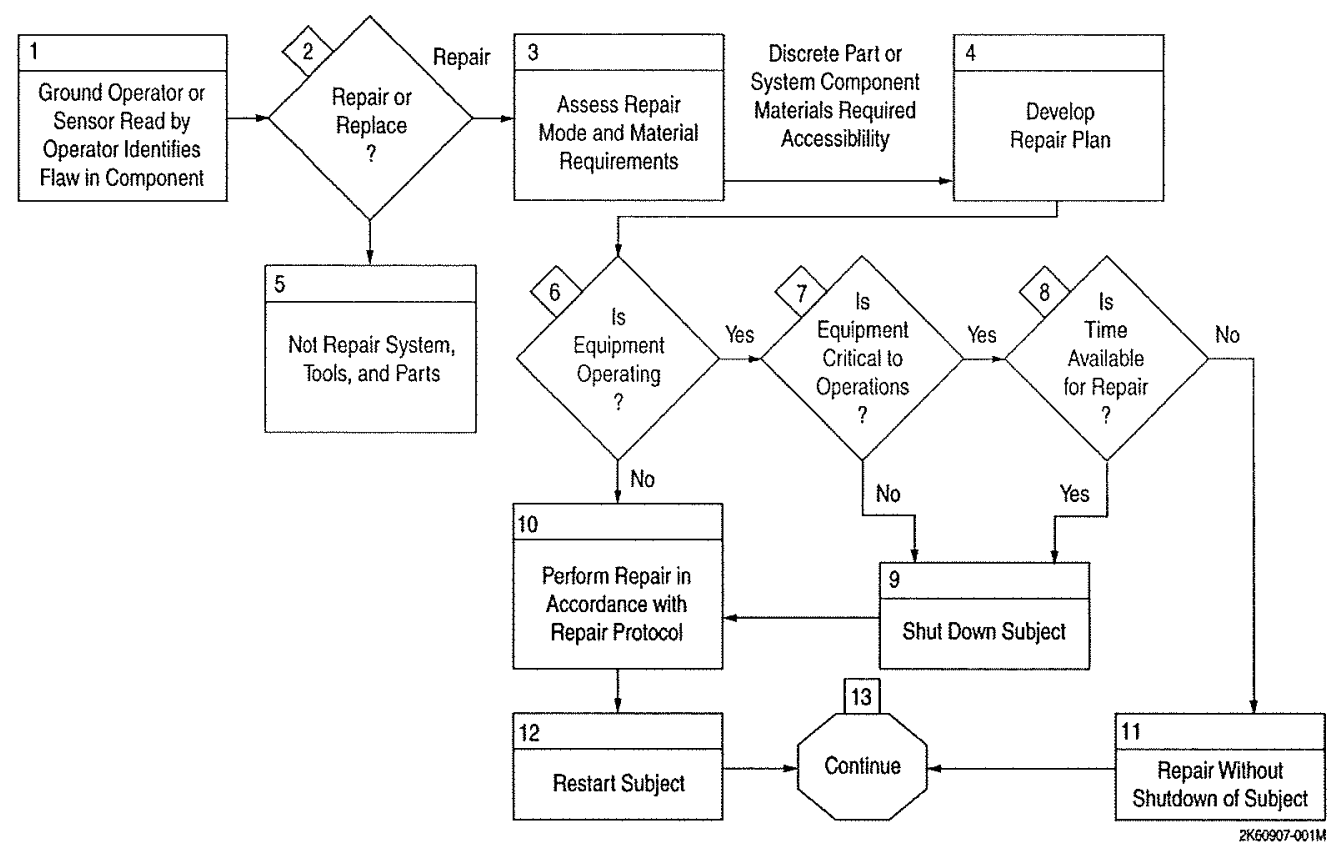

FIGURE 1. Top-Level Repair Scenario.

The analysis of these scenarios also reveals that in the case of emergencies the crew will need to be given a level of autonomy which brings with it a degree of risk. Each step in the repair process will need to be scrutinized for deviations from repair protocol so that the crew and the ground fully understand the risks to be weighed against the level of emergency. As shown in Figure 2 the implementation of autonomous decision making can provide a stop gap for emergencies for a new repair process.

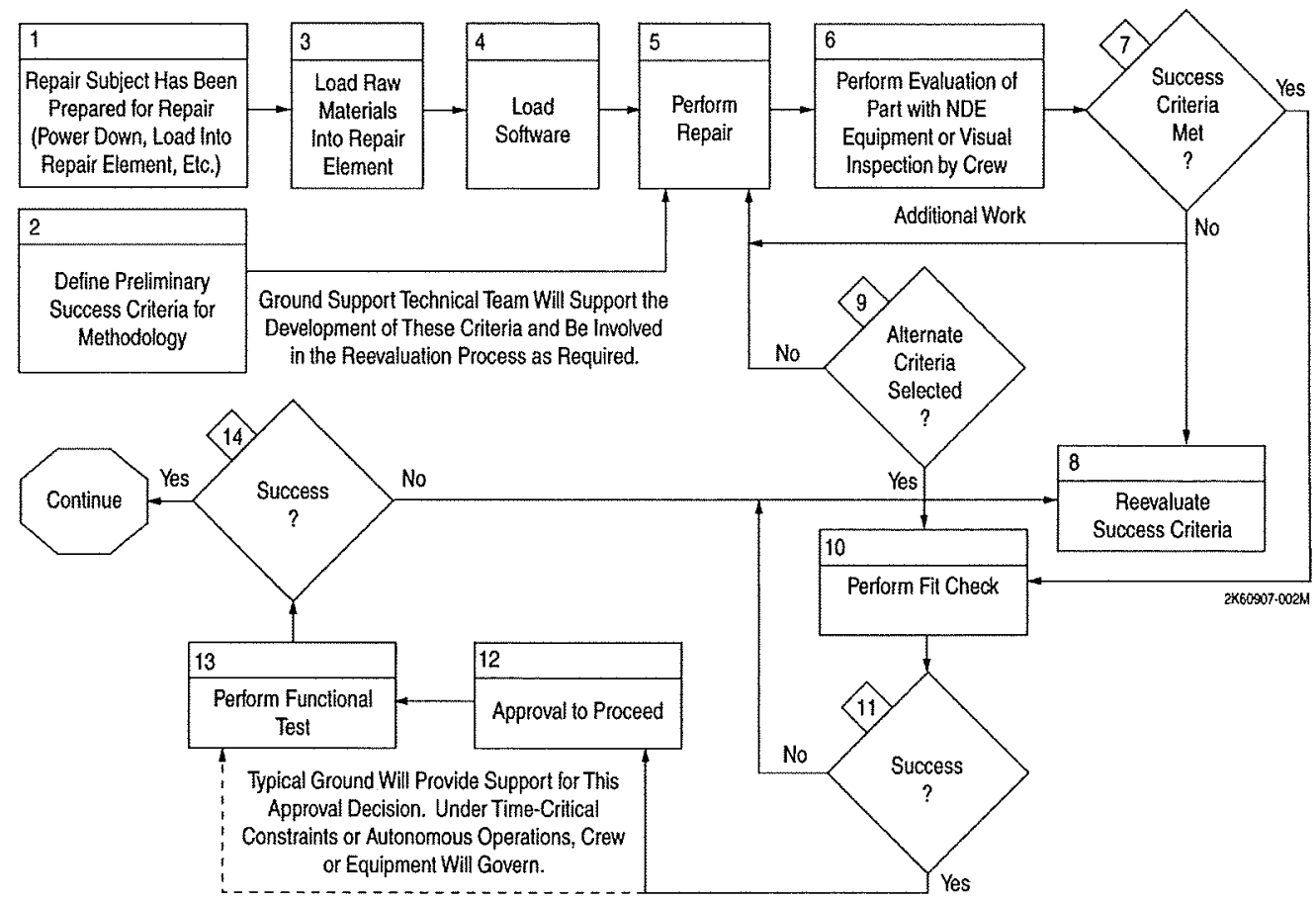

FIGURE 2. New Repair Capability Development and Qualification with Alternate Path for Time-Critical or Emergency Operations. 


\section{DESIGN FOR MAINTAINABILITY AND ACCESSIBILITY}

As noted, traditional approaches have involved component replacement at the ORU level, if replacement was a planned activity at all. Current design policy is to design more for packaging considerations than for maintainability. In order to even make repairs at the component level feasible, new philosophies in design must be acknowledged and embraced.

\section{Design for Access and Ability to Evaluate}

Before a repair solution can be initiated, diagnosis must be possible; in order to perform diagnosis, inspection must be possible. It is important that NDE be taken into consideration early in the design process, and partnerships actively sought with the relevant NDE organizations. In the past, non-destructive evaluation of parts and systems has been, at most, an after-thought. In the process of materials selection during design and development, consideration can be given to what inspection techniques will best yield useful results, and for what materials would render difficult diagnosis of potential repair situations.

In terms of permitting access to necessary parts and systems, design consideration must be given to the ability to inspect and evaluate, as well as remove and reinstall repaired parts. Since candidate repair operations will be more feasible with parts constructed from particular materials, this must also play into design criteria. Of course, ease of access and specific material selection must not be allowed to impact the functionality of the parts or systems, but these considerations should play into the selection process.

In designing to allow for this, it will be important to minimize the crew time that will be needed to perform evaluation, and the degree to which crew are exposed to dangerous operations (e.g. EVA). Among other things, this means maximizing the degree of automation for tasks, and maximizing the extent to which robotic operation (automation or, more likely, tele-operation) can be used to achieve the access. It will also be important to design systems such that the extent of crew training is kept to a minimum. Most methods of non-destructive evaluation require expert interpretation for definitive analysis; to train crew members to this degree is impractical. Instead, infrastructure must be designed to allow signals and images to be transmitted to ground-based experts for analysis, with results quickly relayed to crew.

One method that has been used consistently in the long duration experience such as the International Space Station (ISS) is the use of fabric screens and nets to eliminate concerns from inadvertent contact by a crewmember while providing free access to a tool. This concept may prove to be a good choice for future vehicles where it does not introduce new issues of cleanliness, safety, or fire prevention. The strength of new fabrics is certainly sufficient for the job and the compliance of a fabric boundary will reduce collateral damage to fingers, knuckles and elbows in the maintenance operation.

\section{New Materials}

Interest has been increasing and promising research is underway in the area of self healing materials. These materials ranging from composites to metals could have direct application in design and construction of space vehicles and systems. As greater maturity is achieved, use of such materials will make it possible to greatly reduce or eliminate the need for accessibility to certain sub-systems. Such materials will have to reach considerably higher levels of maturity (i.e. higher TRLs) before it will be possible to incorporate them into designs with a high level of confidence. At current levels of progress and sophistication, it does not appear that any such materials will be ready for consideration in the first generation of Exploration Launch Vehicle systems, such as the Crew Launch Vehicle (CLV) or Crew Exploration Vehicle (CEV). However, these advancements bear watching, and self healing materials have the potential to make significant contributions to the design of later-generation Exploration equipment.

Current efforts are underway for self healing electrical/electronic PC boards and wires. Since electronics of the future are miniature to subminiature the repair techniques require specialized skills on the order of surgery. The extremely compact packaging designs present challenging implications for preventing collateral damage. Self healing composite boards, traces, and solder joints, would reduce the burden on the crew for training and specialized dexterity. Self healing doesn't come without its additional complexities, and it will be important to have sensing that 
identifies that damage and repair has occurred. It would be self defeating to allow repeating failures that self heal to continue and possibly escalate to detrimental levels. This would not be the case for single incident damage from events such as micrometeor impacts.

Self healing insulations for wire bundles provide obvious benefits to future flights. Electrical damage repair to clusters of tightly bound bundles of wire is an arduous task requiring many levels of disassembly with potential to do further damage at each step. Healing insulation and conductor concepts are in work but at very low TRL.

Materials are also a consideration for repairability by conventional means. The concept of selections based on compatibility with adhesives, corrosion resistance, melting points, fuming temperatures, ductility, etc must be infused into the mind of designers. Metals have historically been good choices for adapting to adhesive or mechanical fastener solutions. Resistance to corrosion of the bare metal is an important feature in assuring quality bonds. Alloys that resist immediate oxidation upon exposure will be high in the list of selectors. Thermoplastics are historically difficult to bond using adhesives. Joining techniques where moderate temperature is applied to fuse surfaces is an alternative to introducing adhesives. The use of friction stir welding for metals is a favorable alternative with new hand-held welding tools in development. Fiber reinforced composites (typically thermosetting plastic resins) offer excellent bonding surfaces in damage areas due to the coarse and fibrous interface resulting from fracture or puncture damage. In this case excessive particulates are a concern which may override the benefits of a compatible surface.

Memory shape materials are a relatively new field of application though these materials have been around for generations. These materials can be applied as patches or can be reformed after damage by application of energy such as heating. The strength of these materials when combined with fiber reinforcement is remarkable considering the elevation of temperatures by approximately $50 \mathrm{~K}$ provides a very compliant and flexible working piece. The inventive uses of these memory materials have so far only had cursory investigation.

\section{THERMAL CYCLING}

The long term thermal cycling that will be experienced by components used on the Moon and Mars will be unlike anything that has been experienced in the history of manned space flight. Depending on landing sites chosen, thermal cycling experienced by Lunar equipment could range from about $115 \mathrm{~K}$ to $395 \mathrm{~K}$ (Heiken, Vaniman, French, 1991). Much less is known about surface conditions in the Lunar polar regions, but the temperature variations for most of the surface there are expected to be much smaller, from about $210 \mathrm{~K}$ to $230 \mathrm{~K}$. The Apollo missions avoided these temperature cycling extremes by landing in equatorial regions shortly after local Lunar dawn, and departing well before local Lunar noon. The temperature cycling for Martian missions will be less extreme (on the order of $113 \mathrm{~K}$ to $293 \mathrm{~K}$ ), but will still be substantial.

As equipment is exposed to this "daily" cycle of temperatures (the Lunar day being about four weeks long), it will naturally experience stresses unlike any terrestrial equipment. The day/night swings of $60 \mathrm{~K}$ in the high desert on Earth are small by comparison. Equipment must naturally be designed to withstand these rigors.

The much smaller shifts in temperatures experienced at the Lunar poles will provide no respite for these design constraints. The poles are of high interest primarily because of the tantalizing suggestion of the presence of water ice in the permanently shadowed regions and the possibility of extracting this valuable resource. In the permanently shadowed craters, temperatures are estimated to remain at about $40 \mathrm{~K}$. In order to extract the ice, some equipment, at least, will have to venture into the permanently shadowed regions and back, subjecting it to temperature cycles on the order of $190 \mathrm{~K}$.

When considering the design of materials and equipment for repair of systems operating in these thermal conditions, one must consider the tendency of this transient to crystallize most compatible materials. Differences in thermal coefficients of expansion must be considered as well.

Adhesive-based repairs have shown to be one of the most widely applicable methods for repairs of different types of equipment and for wide ranges of materials. Polymers which can span the temperature ranges to be encountered are available for adhesives, but new constituencies must be developed and tested. At extremely low temperatures, curing 
will also be a substantial issue. Self-sustaining curing can meet this challenge. Combustion synthesis and frontal polymerization can provide a temporary, localized environment with an elevated temperature for the curing process.

Use of like materials for joining will still be available and desirable as in the case of moderate temperature electrical joining. Metal joining for structural materials will require significant process development to eliminate the hazards of high and low temperature exposure; however, these issues are not insurmountable and the hazards will be controllable making metal on metal still a solid solution for permanent repairs.

\section{CONTROL OF MATERIALS AND ENVIRONMENTS}

During the Apollo excursions on the Lunar surface, substantial difficulty was encountered with Lunar dust (National Aeronautics and Space Administration Manned Spacecraft Center, 1970). Lack of familiarity with the reduced gravitation conditions resulted in other material handling difficulties as well. In the intervening years, experience and knowledge have been gained with respect to the effects of microgravity on material handing. While the experience gained in low Earth orbit will largely translate to the low gravity conditions of the Lunar surface (and to the Martian surface beyond that), issues involved in the control of materials will still need to be addressed.

With reduced gravitational fields, other disruptive forces, such as static charge, make Earth-based control techniques less adaptable. Water vapor is (obviously) not present on the surface of the Moon (and only in trace amounts on Mars). Since moisture cohesively binds dust and debris on Earth, and this effect is absent on the Moon and nearly so on Mars, the net effect is the potential for a dust cloud with reduced visibility and reduced control of cleanliness.

The Martian "dust storms" are well documented, and the tendency for dust to be spread forcefully on the Lunar surface from such effects as the exhaust of a landing spacecraft are similarly well known (National Aeronautics and Space Administration Manned Spacecraft Center, 1970). In addition, the dust plumes caused by such activity as the maneuvers of the Lunar Rover are well documented (NASA History Division, 1972). There is debate about the presence of the existence of "clouds" of dust near the Lunar surface, presumably caused by charges imposed at the Lunar dawn. However, from the Apollo experiences, there is no question about the deleterious effects of dust coating and clinging to equipment, including spacesuits (National Aeronautics and Space Administration Manned Spacecraft Center, 1970).

One partial answer is the use of containment curtains and drapes, as often used on Earth. This will undoubtedly be the best answer in some cases. However, the necessity of contact with equipment, especially in unplanned places or in unplanned ways, such as will be the case in contingency scenarios for small repair tasks, will mean that other techniques will need to be pursued. Specific methods for dust containment or exclusion will need to be considered for each repair tool utilized, but this is true for many sorts of operations that will be carried out during EVA on the surface of the Moon and Mars. Devices built into the repair tooling that provide localized volumes to control the environment at the site of the repair are one answer that may ease the burdens of material control.

\section{LOW PRESSURE}

The atmospheric pressure at the Martian surface is less than one percent of that of the Earth at sea level; on the surface of the Moon there exists a state of ultra-high vacuum (less than $10^{-12} \mathrm{kPa}$ ), expensive to replicate in test vacuum chambers, and extremely difficult to replicate for full-scale ground testing. The low atmospheric pressure on the Martian surface will present substantial challenges in and of itself, including the rapid boiling of many common liquids, but the much harsher environment of the Moon will be encountered first.

The cleanliness of prepared surfaces has a substantial impact on the ability of adhesives, for instance, to be applied and utilized successfully. The ultra-high vacuum conditions on the Lunar surface would be sufficient to flash all volatiles normally used for cleaning and preparing surfaces, and this will have to be addressed. Other methods of cleaning or the development of techniques that are not so dependent on prepared (cleaned) surfaces will need to be developed.

The effects of vacuum on the constituent materials used for adhesive repairs themselves are not fully characterized and require further testing. It is expected that the high rates of evaporation may significantly weaken solvent-based 
material repairs. Formation of bubbles presents porosity issues. Accelerated curing may lead to an incomplete cure and result in reduced bonding strength for conventional adhesive approaches. These issues need to be addressed or eliminated.

One approach that has shown some early promise is the use of adhesives based on ionic liquid solutions. The use of these chemicals which exhibit extremely low vapor pressures makes possible the tailoring of adhesive agents that provide extremely stable low pressure characteristics. Another approach to mitigate or eliminate the difficulties of adhesive repairs in vacuum is to use quick-curing adhesives not based on solvents, using such methods as heat, ultra-violet or visible-light initiated curing methods. Simple tools such as light pens can be used to locally initialize the curing.

A new material which has the characteristic of solidifying when electric current is passed through it provides another opportunity to establish a containment boundary. As a concept, the electro curing adhesive could be used as a sealer for a containment capsule used to isolate the repair during the curing period. Following the repair the current is discontinued and the electro adhesive debonds with the surface and the capsule is removed with no permanent residue.

It should be noted that the vacuum of the Lunar surface does provide one substantial benefit: the near-elimination of oxidation, a serious problem for terrestrial joining repairs. However, this is but one aspect of a situation that will provide substantial environmental challenges.

The low pressure environment provides another opportunity which is compatible with the low level of dexterity typical of EVA gloves. In conventional adhesive application, the operator provides the force to the plunger which dispenses the adhesive. In the low pressure environment the operator need only apply the activation of an open/close orifice which through the use of the pressure differential will dispense the adhesive at a controlled rate. The activation could even be controlled by the crewmember's mouth via microswitch or pneumatic toggle. The crewmember can concentrate his hand dexterity efforts to positioning the application tool.

\section{LIMITED DEXTERITY AND MANEUVERABILITY}

Conventional designs of space suits, such as that of the Apollo A7L and A7L-B suits, the current NASA Extravehicular Mobility Unit (EMU) and the Russian Orlan space suits, place severe limits on the maneuverability, mobility and dexterity of the wearer in any EVA. Indeed, while other concepts for space suits have been proposed, such as the mechanical counterpressure suits tested by NASA in the early 1970s, all suits that have been used in the harsh environment of space to date have been of designs that severely limited maneuverability.

Of particular concern is the lack of manual dexterity found in manipulating the gloves of these suits. Fine motor skills, including the gripping of small objects, are very difficult to replicate in these suits. As a consequence, concepts of tools have been designed with the limited abilities of the wearer in mind: large grips, large handles and long levers. An example is the repair tool prototype in NASA's Kit for External Repair of Module Impacts (KERMIt), developed for dispensing adhesives to seal punctures on the International Space Station.

In addition, it is acknowledged that long duration activity in these suits can be very fatiguing. Even the amount of time that the Apollo astronauts spent on the Lunar surface proved to cause physical hardship (National Aeronautics and Space Administration Manned Spacecraft Center, 1971). In recognition of the need for new concepts and new designs, NASA is already soliciting industry to assist in the planning for a new design of a space suit system (NASA Johnson Space Center (JSC) Procurement, 2006).

Design of repair tools and procedures will have to take these physical limitations into consideration. (This must also be a consideration in the design of the systems that might have to be repaired in an EVA operation; as noted above, concepts for repairability must be examined from early stages in design to make later repairs feasible.) More ergonomic designs of the repair tools will incorporate pushbutton controls and simplified visual cue electronic displays to minimize the dexterity requirements. 


\section{IMPLEMENTATION OF VERIFICATION REQUIREMENTS}

Aside from the performance aspects of in situ repair comes the administrative issue of implementing a verification program for repairs. This challenge encompasses and surpasses the performance issues. As with all space flight the demand for minimum risk to crew and equipment will be necessary and uncompromising. Since repair is such a unique operation it is unrealistic to attempt to qualify every possible scenario, and what is possible in verifying equipment manufactured on the ground is not possible for equipped repaired while in space. What is possible is to fully understand the available repair processes so that application specific repairs could be evaluated prior to and (with advanced tools and techniques) following the repair process. Table 1 provides some of the issues that must be overcome to meet the in situ verification challenge.

TABLE 1. Issues with Verification Program for In Situ Repairs.

\begin{tabular}{lll}
\hline Challenge & Ground Verification & In Situ Verification \\
\hline Test equipment availability & $\begin{array}{l}\text { Extensive equipment and facilities } \\
\text { available at NASA centers, } \\
\text { universities, and industry }\end{array}$ & $\begin{array}{l}\text { Limited to payload or habitat } \\
\text { provisions }\end{array}$ \\
\hline $\begin{array}{l}\text { Test and Quality Assurance } \\
\text { personnel }\end{array}$ & $\begin{array}{l}\text { Extensive sources for personnel and } \\
\text { expertise. }\end{array}$ & $\begin{array}{l}\text { Limited to crew and data that can be } \\
\text { downlinked for evaluation. }\end{array}$ \\
\hline Protection and safety during testing & $\begin{array}{l}\text { Ground activities can utilize } \\
\text { extensive space for isolation of } \\
\text { hazardous testing to prevent hazards } \\
\text { to test personnel }\end{array}$ & $\begin{array}{l}\text { Space and isolation are limited by } \\
\text { vehicle and habitats }\end{array}$ \\
\hline Analysis capabilities & $\begin{array}{l}\text { Extensive sources for personnel and } \\
\text { expertise }\end{array}$ & $\begin{array}{l}\text { No significant differences; however, } \\
\text { longer time delays for transmission } \\
\text { of information will occur because of } \\
\text { transmission coverage }\end{array}$ \\
\hline $\begin{array}{l}\text { Accessibility of hardware for } \\
\text { functional evaluation }\end{array}$ & $\begin{array}{l}\text { Ground units or prototypes are } \\
\text { usually available for integrated } \\
\text { checkout prior to installation into the } \\
\text { flight unit, reducing risk to critical } \\
\text { equipment }\end{array}$ & $\begin{array}{l}\text { Integration into the flight hardware } \\
\text { may be the only means for } \\
\text { functional evaluation in the } \\
\text { integrated assembly }\end{array}$ \\
\hline
\end{tabular}

\section{CONCLUSIONS}

To enable the long duration missions that are necessary for fulfilling the president's Vision for Space Exploration, necessary launch masses must be minimized for space missions. To accomplish this, new paradigms for the overall concept of mission strategy must be adopted, including designing systems with repairability in mind. However, several issues must be addressed and conquered before the idea of making systems and parts repairable in space becomes reality.

Each of the issues presented herein can be overcome with the application of a thoughtful investigation of methods from the past integrated with technologies of the future. The effective coordination and communication of research and technology advancements among the various Constellation Program areas is a necessity in order to fully capitalize on available technology and reap maximum benefits. NASA schedules and budgets are tight, so it is imperative that consideration be given to designing in approaches for "repairability" early in the hardware design phase. The return on this "repairability" investment in hardware will be realized in terms of fewer spares being manufactured and launched translating into lower costs, more upmass availability for payload, and most importantly the ability to be self-sufficient while away from Mother Earth. 


\section{ACKNOWLEDGMENTS}

Portions of this work were performed in support of the Marshall Space Flight Center under the Microgravity Science and Applications Department (MSAD) Systems Development and Operations Support (SDOS) Contract (No. NA.S8-02060).

\section{REFERENCES}

Bodiford, M.P., Gilley, S.D, Howard, R.W., Kennedy, J.P. and Ray, J.A., "Are We There Yet? ... Developing In Situ Fabrication

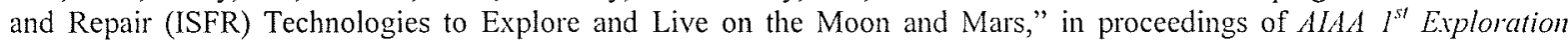
Conference, American Institute of Aeronautics and Astronautics, Reston, VA, 2005, AIAA 2005-2624.

Hammond, M.S., Good, J.E., Gilley, S.D. and Howard, R.W., "Developing Fabrication Technologies to Provide On Demand Manufacturing for Exploration of the Moon and Mars," in proceedings of $44^{\text {th }}$ AIAA Aerospace Sciences Meeting and Exhibit, American Institute of Aeronautics and Astronautics, Reston, VA, 2006, AIAA 2006-526.

Heiken, G.H., Vaniman, D.T. and French, B.M., editors, Lunar Sourcebook: A User's Guide to the Moon, Cambridge University Press, Cambridge, UK, 1991, p. 36.

NASA History Division, (1972), http:/www.hq.nasa.gov/office/pao/History/40thann/mpeg/ap16_rover.mpg, accessed 31 August, 2006.

NASA Johnson Space Center (JSC) Procurement, Constellation Space Suit System (CSSS) Contract, (2006), http://procurement.jsc.nasa.gov/csss/, accessed 03 August, 2006.

National Aeronautics and Space Administration Manned Spacecraft Center, NASA SP-238, "Apollo 11 Mission Report," (1971), http://ntrs.nasa.gov/archive/nasa/casi.ntrs.nasa.gov/19710015566_1971015566.pdf, accessed 31 August, 2006.

National Aeronautics and Space Administration Manned Spacecraft Center, "Apollo 12 Mission Report," (1970), http://ntrs.nasa.gov/archive/nasa/casi.ntrs.nasa.gov/19760072997_1976072997.pdf, accessed 31 August, 2006.

Mankins, J.C., NASA, Office of Space Access and Technology, Advanced Concepts Office, "Technology Readiness Levels: A White Paper," (1995), http://www.hq.nasa.gov/office/codeq/trl/trl.pdf, accessed 31 August, 2006. 